\title{
On Quasi Medians of a Weibull Sample
}

Ahmed Zogo Memon

Visiting Faculty

College of Statistical and Actuarial Sciences

University of the Punjab, Lahore, Pakistan

azogomemon@hotmail.com

Abdur Razaq

National College of Business Administration \& Economics

Lahore, Pakistan

razaqfb18@yahoo.com

\section{Abstract}

A sample quasi median may be used to estimate the median of a distribution when information on the sample median is not available. This paper expresses moments of Quasi medians of a Weibull sample in terms of incomplete beta functions. A comparison of quasi medians and sample median is made for the purpose of estimating the median of Weibull distribution when its shape parameter assumes various values.

Keywords: Weibull order statistics; Sample Median and Quasi Median.

\section{Introduction}

Let $Y_{1}, Y_{2}, \ldots Y_{n}$ be the order statistics based on a random sample of size $n$ from the Weibull distribution (1951) introduced by Wallodi Weibull (1887-1979)

$$
F(x)=1-\exp \left(-\alpha x^{\beta}\right), \quad x>0,
$$

with $\alpha, \beta>0$. Memon and Daghel (1987) investigate the sampling distribution of

$$
Z=a\left(Y_{t}\right)^{c}+b\left(Y_{m}\right)^{d}, \quad 1 \leq t \leq m \leq n,
$$

for some special cases where the constants $a, b>0 ; c, d$ are known real numbers. Memon $(1987,2008)$ finds expressions for moments of linear combination of powered order statistics in terms of incomplete Beta function $\beta_{p}(\mu, v)$, and examine the behaviour of the powered sample median and some other order statistics suggesting the values of $c / \beta$ that provide near symmetrical distributions.

Hodges \& Lehmann (1967) discuss sample medians and quasi medians in their paper [2] and derive the efficiencies of median relative to that of sample mean. For a random sample of an odd size $n=2 v+1$ the median $Z_{M}$ of a distribution 
is often estimated by the sample median $Y_{(v+1)} ; v$ being a positive integer. The $(v-m+1)$ th quasi median of a sample is measured by

$$
Z_{(v-m+1)}=\left(Y_{(m)}+Y_{(2 v-m+2)}\right) / 2 ; \quad m=1,2, \ldots, v
$$

The quasi medians in the neighbourhood of $Y_{(v+1)}$ can also offer estimates of $Z_{M}$ with varying efficiencies. When the information to find $Y_{(v+1)}$ is damaged, or distorted and so assumed to be unavailable, the first quasi median $Z_{(v)}$ that is closest to $Y_{(v+1)}$, naturally becomes a preferable substitute for the sample median to estimate $Z_{M}$.

In this paper we attempt to evaluate the relative performance of quasi medians to that of sample median using a random sample of size $n=2 v+1$ from Weibull distribution; realizing that $\beta$ and $n$ are both important for this purpose. The case when $n$ is even is developed similarly without proof.

\section{Lemmas}

\section{Lemma 1}

a) $\quad \sum_{i=1}^{n}\left(\begin{array}{c}n-1 \\ i-1\end{array}\right)(-1)^{i-1}(1+i)^{-1}=\frac{1}{n(n+1)}$

b) $\quad \sum_{i=1}^{n}\left(\begin{array}{c}n-1 \\ i-1\end{array}\right)(-1)^{i-1}(i)^{-1}=\frac{1}{n}$

Proof For (a) the left side can be written in the form

$$
\left[n^{-1} \sum_{i=1}^{n}\left(\begin{array}{l}
n \\
i
\end{array}\right)(-1)^{i-1}-[n(n+1)]^{-1} \sum_{i=1}^{n}\left(\begin{array}{c}
n+1 \\
i+1
\end{array}\right)(-1)^{i-1}\right]
$$

from which the result follows.

For (b) the proof is trivial.

Lemma 2 Let $Y_{t}, Y_{t+1}$ be the Weibull order statistics. Then

$$
\begin{aligned}
& \begin{array}{l}
E\left(Y_{t} Y_{t+1}\right)=\gamma \sum_{i=1}^{t}\left(\begin{array}{c}
t-1 \\
i-1
\end{array}\right)(-1)^{i-1}\left(\gamma_{i 1}\right)^{-(1 / \beta)-1}\left(\gamma_{1}\right)^{-(1 / \beta)-1} \beta_{p}[(1 / \beta)+1,(1 / \beta)+1] \\
\text { where } \quad p=\gamma_{i 1} /\left(\gamma_{1}+\gamma_{i 1}\right), \\
\qquad \gamma=n ![(t-1) !(n-t-1) !]^{-1} \Gamma((2 / \beta)+2)
\end{array}
\end{aligned}
$$




$$
\begin{aligned}
& \gamma_{i 1}=i \\
& \gamma_{1}=(n-t)
\end{aligned}
$$

and $\beta_{p}[(1 / \beta)+1,(1 / \beta)+1]$ is an incomplete Beta function.

The proof follows by taking appropriate values in Theorem I, Sec. 3.2 Memon (2008).

\section{Distribution of $Z_{(v-m+1)}$}

$$
(n=2 v+1)
$$

We determine here the distribution of the quasi median $Z_{(v-m+1)}$. The joint pdf of the two Weibull order statistics $\left(Y_{(m)}, Y_{(2 v-m+2)}\right)$ can be expressed as

$$
\begin{gathered}
\phi \beta^{2} \sum_{l=1}^{m} \sum_{j=1}^{2 v-2 m+2}\left(\begin{array}{c}
m-1 \\
l-1
\end{array}\right)\left(\begin{array}{c}
2 v-2 m+1 \\
j-1
\end{array}\right)(-1)^{l+j}\left[\left(y_{(m)} y_{(2 v-m+2)}\right)\right]^{\beta-1} \\
{\left[\exp \left\{-\gamma_{m j l}\left(y_{(m)}\right)^{\beta}-\gamma_{m j}\left(y_{(2 v-m+2)}\right)^{\beta}\right]\right.} \\
0<y_{(m)}<y_{(2 v-m+2)} \\
m=1,2, \ldots, v
\end{gathered}
$$

where

$$
\begin{aligned}
& \phi=(2 v+1) !\left[((m-1) !)^{2}(2 v-2 m+1) !\right]^{-1} \\
& \gamma_{m j}=m+j-1, \gamma_{m j l}=2 v-2 m+l-j+2
\end{aligned}
$$

To find the distribution of $Z_{(v-m+1)}$ let

$Z=Y_{(m)}+Y_{(2 v-m+2)}$

$W=Y_{(m)}$

so that the pdf of $(Z, W)$ is $h(z, w)=$

$$
\begin{gathered}
\phi \beta^{2} \sum_{l=1}^{m} \sum_{j=1}^{2 v-2 m+2}\left(\begin{array}{c}
m-1 \\
l-1
\end{array}\right)\left(\begin{array}{c}
2 v-2 m+1 \\
j-1
\end{array}\right)(-1)^{l+j}[(w)(z-w)]^{\beta-1}\left[\exp \left\{-\gamma_{m j l}(w)^{\beta}-\gamma_{m j}(z-w)^{\beta}\right]\right. \\
0<w<z<\infty
\end{gathered}
$$


The distribution of $Z$, and so that of $Z_{(v-m+1)}$, follows as

$$
h\left(z_{(v-m+1)}\right)=2 \int_{0}^{2 z_{(v-m+1)}} h(2 z, w) d w
$$

\subsection{Moments of $Z_{(v-m+1)}$}

We now state and prove the following theorem on the moments of $Z_{(v-m+1)}$ for the case when $(n=2 v+1)$.

THEOREM The $r$-th moment of Weibull quasi median $Z_{(v-m+1)}$ is given by $\gamma \sum_{l=1}^{m} \sum_{j=1}^{2 v-2 m+2} \sum_{k=0}^{r}\left(\begin{array}{l}r \\ k\end{array}\right)\left(\begin{array}{l}m-1 \\ l-1\end{array}\right)\left(\begin{array}{c}2 v-2 m+1 \\ j-1\end{array}\right)(-1)^{l+j}\left[\left(\gamma_{m j l}\right)^{-\frac{k}{\beta}-1}\left(\gamma_{m j}\right)^{-\frac{r-k}{\beta}-1} \beta_{p}\left\{\frac{k}{\beta}+1, \frac{r-k}{\beta}+1\right\}\right]$

where

$$
\begin{aligned}
& \gamma_{m j}=m+j-1, \\
& \gamma_{m j l}=2 v-2 m+l-j+2, \\
& \gamma=(1 / 2)^{r}(2 v+1) !\left[((m-1) !)^{2}(2 v-2 m+1) !\right]^{-1} \Gamma((r / \beta)+2) . \\
& p=\gamma_{m j l} /\left(\gamma_{m j}+\gamma_{m j l}\right)
\end{aligned}
$$

$\beta_{p}[(k / \beta)+1,((r-k) / \beta)+1]$ is an incomplete Beta function.

Proof From Eq,(3.1) the $r$-th moment of $Z_{(v-m+1)}$ is obtained from

$$
(2)^{-r} \iint z^{r} h(z, w) d w d z
$$

which can be simplified to

$$
\begin{gathered}
(2)^{-r} \phi \beta^{2} \sum_{l=1}^{m} \sum_{j=1}^{2 v-2 m+2} \sum_{k=0}^{r}\left(\begin{array}{l}
r \\
k
\end{array}\right)\left(\begin{array}{c}
m-1 \\
l-1
\end{array}\right)\left(\begin{array}{c}
2 v-2 m+1 \\
j-1
\end{array}\right)(-1)^{l+j} \iint[(w)]^{k+\beta-1}[(z-w)]^{r-k+\beta-1} \\
\quad\left[\exp \left\{-\gamma_{m j l}(w)^{\beta}-\gamma_{m j}(z-w)^{\beta}\right] d w d z\right. \\
0<w<z<\infty
\end{gathered}
$$

The expression under integration comprises two terms separated by a minus sign. We consider the integral

$$
\iint[(w)]^{k+\beta-1}[(z-w)]^{r-k+\beta-1}\left[\exp \left\{-\gamma_{m j l}(w)^{\beta}-\gamma_{m j}(z-w)^{\beta}\right\}\right] d w d z
$$


On using the transformation

$$
\begin{aligned}
& v_{1}=\gamma_{m j l}(w)^{\beta} \\
& v_{2}=\gamma_{m j}(z-w)^{\beta}
\end{aligned}
$$

and the relevant Jacobian we get

$$
\beta^{-2}\left(\gamma_{m j l}\right)^{-(k / \beta)-1}\left(\gamma_{m j}\right)^{-(r-k) / \beta-1} \iint v_{1}^{k / \beta} v_{2}^{(r-k) / \beta}\left[\exp \left[\left(-v_{1}-v_{2}\right)\right] d v_{1} d v_{2}\right.
$$

defined over $\left(v_{1}, v_{2}\right)$ plane such that $0<v_{1}<[p /(1-p)] v_{2}$ and $p>0$. On introducing two new variables one for $v_{1}+v_{2}$ and the other for $v_{1}$, the double integral by Lemma 1 of Memon (2008), simplifies to

$$
\Gamma((r / \beta)+2) \beta_{p}[(k / \beta)+1,((r-k) / \beta)+1]
$$

and so Eq (3.1.4) comes to

$$
\beta^{-2}\left(\gamma_{m j l}\right)^{-(k / \beta)-1}\left(\gamma_{m j}\right)^{-(r-k) / \beta-1} \Gamma((r / \beta)+2) \beta_{p}[(k / \beta)+1,((r-k) / \beta)+1]
$$

and now these substitutions in Eq.(3.1.2) lead to the main result .

3.2 Corollary -1: For $m=1$, the $r$-th moment of the last quasi median $Z_{(1)}$ based on a random sample from Wibull distribution s given by

$$
\gamma \sum_{j=1}^{2 v} \sum_{k=0}^{r}\left(\begin{array}{l}
r \\
k
\end{array}\right)\left(\begin{array}{c}
2 v-1 \\
j-1
\end{array}\right)(-1)^{j-1}\left[\left(\gamma_{v j}\right)^{-\frac{k}{\beta}-1}\left(\gamma_{j}\right)^{-\frac{r-k}{\beta}-1} \beta_{p}\left\{\frac{k}{\beta}+1, \frac{r-k}{\beta}+1\right\}\right]
$$

where

$$
\begin{aligned}
& \gamma_{j}=j \\
& \gamma_{v j}=2 v-j+1 \\
& \gamma=(1 / 2)^{r}(2 v+1) ![(2 v-1) !]^{-1} \Gamma((r / \beta)+2) . \\
& p_{j}=(2 v-j+1) /(2 v+1) \\
& \beta_{p_{j}}[(k / \beta)+1,((r-k) / \beta)+1] \text { is an incomplete Beta function. }
\end{aligned}
$$


3.3 Corollary -2: For $m=v$, the $r$-th moment of the first Weibull quasi median $Z_{(v)}$ is given by

$$
\gamma \sum_{l=1}^{v} \sum_{j=1}^{2} \sum_{k=0}^{r}\left(\begin{array}{l}
r \\
k
\end{array}\right)\left(\begin{array}{l}
v-1 \\
l-1
\end{array}\right)(-1)^{l+j}\left[\left(\gamma_{l j}\right)^{-\frac{k}{\beta}-1}\left(\gamma_{j}\right)^{-\frac{r-k}{\beta}-1} \beta_{p}\left\{\frac{k}{\beta}+1, \frac{r-k}{\beta}+1\right\}\right]
$$

where

$$
\begin{aligned}
& \gamma_{j}=v+j-1, \\
& \gamma_{l j}=l-j+2, \\
& \gamma=(1 / 2)^{r}(2 v+1) ![(v-1) !]^{-2} \Gamma((r / \beta)+2) . \\
& \quad p_{j}=(l-j+2) /(l+r+1)
\end{aligned}
$$

$\beta_{p_{j}}[(k / \beta)+1,((r-k) / \beta)+1]$ is an incomplete Beta function.

Remark: For the first moment of $Z_{(v)}$ we have, $p_{1}=(l+1) /(l+2)$, $p_{2}=l /(l+2)$ in the above corollary. On substituting these values for the incomplete beta functions we find the following coefficient of $(v+l+1)^{-(1 / \beta)-1}$

$$
((1 / \beta)+1)^{-1}\left\{[(v)(v+1)]^{-1}-[(l)(l+1)]^{-1}\right\} .
$$

The other term with $((1 / \beta)+1)^{-1}(v+l+1)^{-(1 / \beta)-1}$ is

$$
v^{-(1 / \beta)-1}(l+1)^{-1}-(v+1)^{-(1 / \beta)-1}(l)^{-1}
$$

By Lemma 1 it can be now verified that $E\left(Z_{(v)}\right)=\left[E\left(Y_{(v)}\right)+E\left(Y_{(v+2)}\right)\right] / 2$.

\section{Moments of $Z_{(v-m)}$}

$$
(n=2 v)
$$

For the case when $n$ is even the sample median and quasi medians are

$$
\begin{aligned}
& Z_{(v)}=\left(Y_{(v)}+Y_{(v+1)}\right) / 2 \\
& Z_{(v-m)}=\left(Y_{(v-m)}+Y_{(v-m+1)}\right) / 2 ; \quad m=1,2, \ldots, v-1
\end{aligned}
$$

respectively. We state here the following theorem. 
THEOREM The $r$-th moment of Weibull quasi median $Z_{(v-m)}$ (even) is given

by

$\gamma \sum_{l=1}^{v-m} \sum_{j=1}^{2 m+1} \sum_{k=0}^{r}\left(\begin{array}{l}r \\ k\end{array}\right)\left(\begin{array}{c}v-m-1 \\ l-1\end{array}\right)\left(\begin{array}{c}2 m \\ j-1\end{array}\right)(-1)^{l+j}\left[\left(\gamma_{m j l}\right)^{-\frac{k}{\beta}-1}\left(\gamma_{j}\right)^{-\frac{r-k}{\beta}-1} \beta_{p}\left\{\frac{k}{\beta}+1, \frac{r-k}{\beta}+1\right\}\right]$

where

$$
\begin{gathered}
\gamma_{j}=j \\
\gamma_{m j l}=2 m-j+l+1, \\
\gamma=(1 / 2)^{r}(2 v) !\left[((v-m-1) !)^{2}(2 m) !\right]^{-1} \Gamma((r / \beta)+2) . \\
p=(2 m-j+l+1) /(2 m+l+1) \\
\beta_{p}[(k / \beta)+1,((r-k) / \beta)+1] \text { is an incomplete Beta function. }
\end{gathered}
$$

From this theorem we can draw results similar to corollaries 3.1 and 3.2.

\section{Comparison of the First and Last Sample Quasi Medians}

We consider here the first and last quasi medians of Weibull samples of an odd size and compare them with respect to their expected values. For this purpose we select three sample sizes $n=7,11,25$. By corollaries in Section 4 we find $E\left(Z_{(v)}\right)$ and $E\left(Z_{(1)}\right)$ for various $\beta$ values.. These graphs, and the others, are produced below by using Tables 1-7 in Appendix.

Fig 1

FIRST QUASI MEDIAN $E\left(Z_{(v)}\right)$

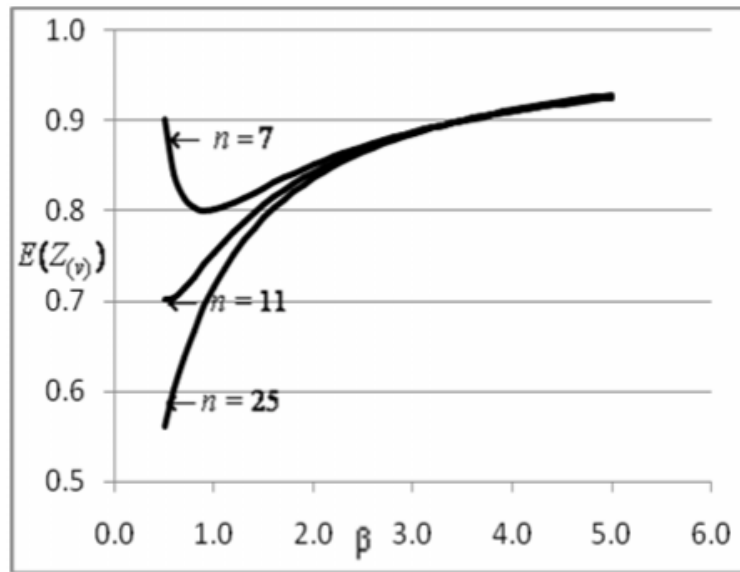

Fig 2

LAST QUASI MEDIAN $E\left(Z_{(1)}\right)$

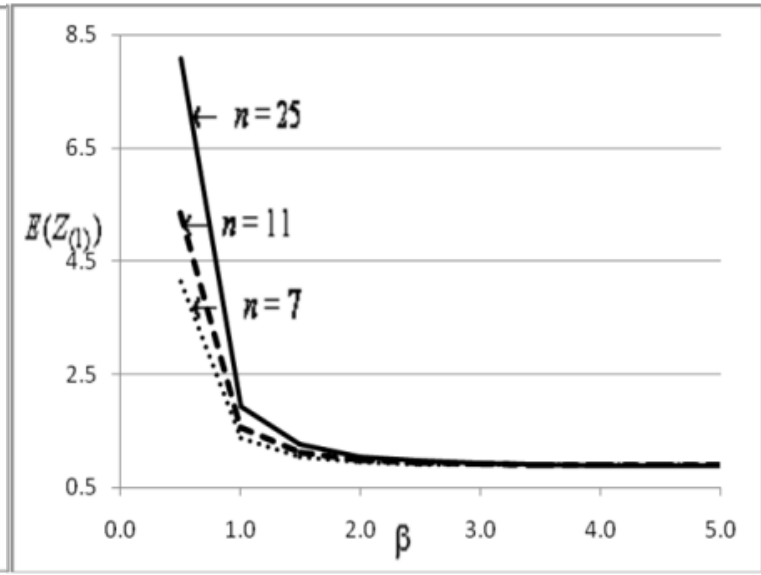

It follows from the above graphs that the two sample medians exhibit much deviation for small values of $\beta$, and that as the sample size increases the expected value of the first (last) quasi median decreases (increases). This pattern occurs for each $\beta$. 
Based on this behavior we therefore assess the comparative performance of the first sample quasi median relative to that of the sample median in the next section.

\section{Performance of First Sample Quasi Median}

In this section we compare for the case $n=2 v+1$ the performance of the sample quasi median $Z_{(v)}$ with that of $Y_{(v+1)}$ for the estimation of the Weibull median $Z_{M}$. It is well known that the r-th moment of $Y_{(v+1)}$ is

$$
\eta \sum_{i=1}^{v+1}\left(\begin{array}{c}
v \\
i-1
\end{array}\right)(-1)^{i-1}(v+i)^{-r / \beta-1}
$$

where $\eta=(2 v+1) ![(v) !]^{-2} \Gamma((r / \beta)+1)$.

6.1 Comparison of their expected values: We present below the graphs of $E\left(Y_{(v+1)}\right), E\left(Z_{(v)}\right)$ based on two sample sizes $n=3, n=25$. A graph for $Z_{M}$ is also included against various values of $\beta$.

Fig 3

For $n=3$

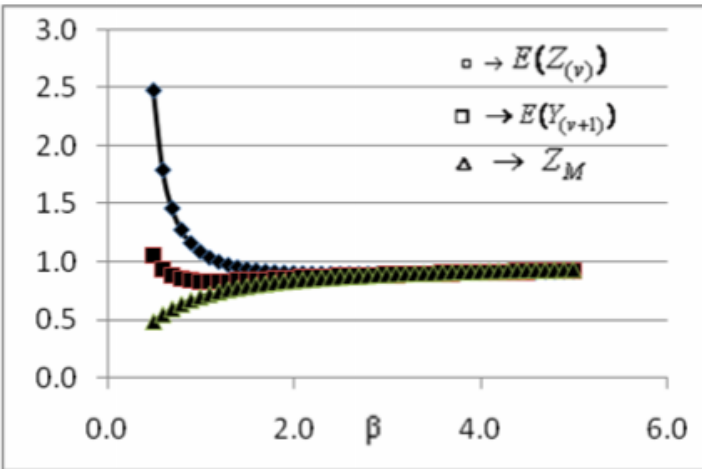

\section{Fig 4}

For $n=25$

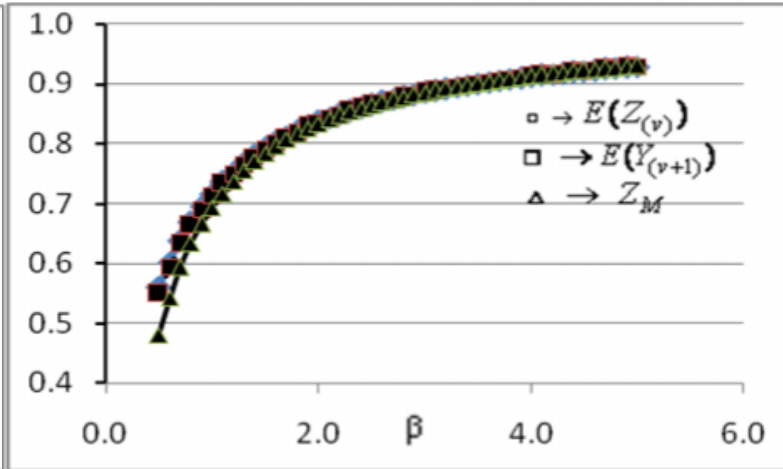

The graphs reveal interesting information regarding these estimators of the Weibull median. The sample median and quasi median overestimate the Weibull median $Z_{M}$ for each value of $\beta$. Each median produces positive bias, and its amount depends both on $\beta$ and $n$. As $\beta$ increases beyond 1.5 the overestimation gradually fades away. The bias due to sample median remains less than that due to quasi median. In this regard, the quantity

$$
\tau=E\left(Y_{(v+1)}-Z_{(v)}\right)
$$

can display a direct comparison of these estimators. The following graphs based on $n=3,11,25$ show a more meaningful picture of this comparison. The smaller 
the absolute value of $\tau$ is the larger the assurance that the quasi median can be used as an option, a useful substitute for the sample median.

$$
\beta, \tau \text { Graph } \quad \text { Fig } 5
$$

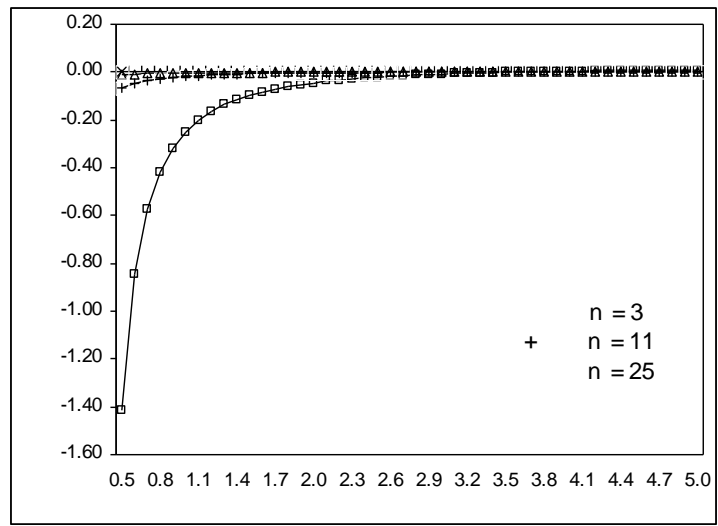

The absolute value of $\tau$ decreases as $\beta$ increases for all sample sizes. For Weibull distributions with small $\beta$ we cannot recommend the use of sample quasi median specially when the sample size is small, but for $\beta \geq 1$ the difference between the two expected values starts rapidly declining. For $\beta \geq 3.5$ it becomes almost negligible.

\subsection{Mean Squared Errors of the Two Sample Medians}

We consider now the mean squared errors $E\left[\left(Z_{(v)}-Z_{M}\right)^{2}\right]$ and $\left.E\left(Y_{(v+1)}-Z_{M}\right)^{2}\right]$ caused by the sample medians $Z_{(v)}$ and $Y_{(v+1)}$ and for it we present the graphs in Fig-6 and Fig-7 relating to $n=3,25$.

Fig 6

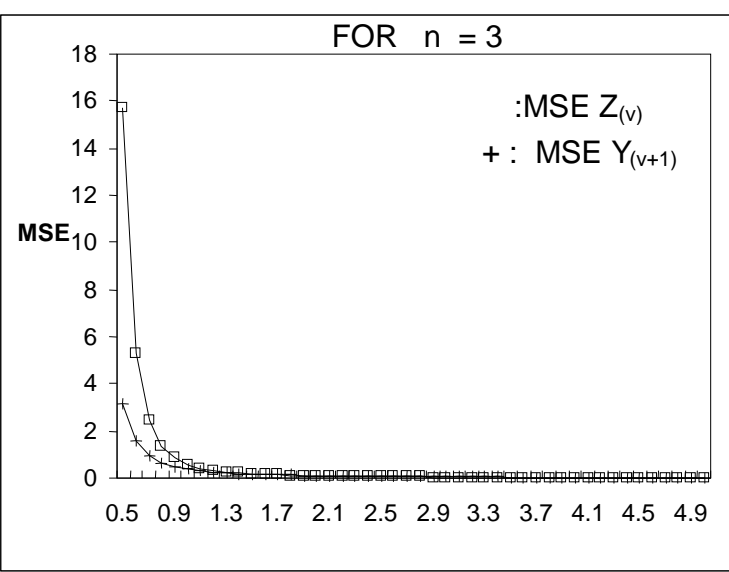

Fig 7

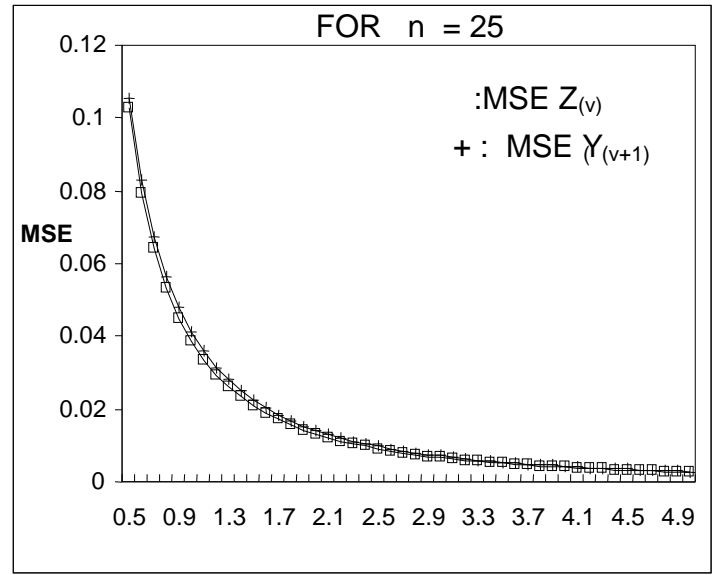

The MSE $\left(Y_{(v+1)}\right)$ remains less than the $\operatorname{MSE}\left(Z_{(v)}\right)$ for both sample sizes. However, for $\beta \geq 1$ the sample quasi median $Z_{(v)}$ serves as an equally good 
estimator for the Weibull median. When $n$, or $\beta$, increases the gap between the two MSEs decreases rapidly.

To see how fast this gap between $Y_{(v+1)}$ and $Z_{(v)}$ decreases with $\beta$, we consider

$$
\xi=E\left(Y_{(v+1)}-Z_{(v)}\right)^{2}
$$

The smaller this quantity is the less risky it is to substitute the sample quasi median for the sample median. The quantity $\xi$ depends on $\beta, n, v$. For its evaluation we need the terms

$$
E\left(Y_{(v+1)}\right)^{2}, E\left(Z_{(v)}\right)^{2} \text { and } E\left(Y_{(v+1)} \cdot Z_{(v)}\right) \text {. }
$$

The first quantity is found from Eq 6.1. The second term follows on taking $r=2$ in the above theorem. And for the third term we can write

$$
2 E\left(Y_{(v+1)} \cdot Z_{(v)}\right)=E\left(Y_{(v)} \cdot Y_{(v+1)}\right)+E\left(Y_{(v+1)} \cdot Y_{(v+2)}\right)
$$

For the calculation of these expected values we take the joint pdf of the two indicated Weibull order statistics and find the expectation of their product. This expectation takes the form of a series involving incomplete beta functions.

Alternatively, by Lemma II, we can find the expectations

$$
E\left(Y_{(v)} \cdot Y_{(v+1)}\right) \text { and } E\left(Y_{(v+1)} \cdot Y_{(v+2)}\right)
$$

so that $\xi$ in 6.2 .1 can now be determined.

We compute the values of $\xi$ and obtain the following graph:

$$
\beta, \xi \text { Graph Fig } 8
$$

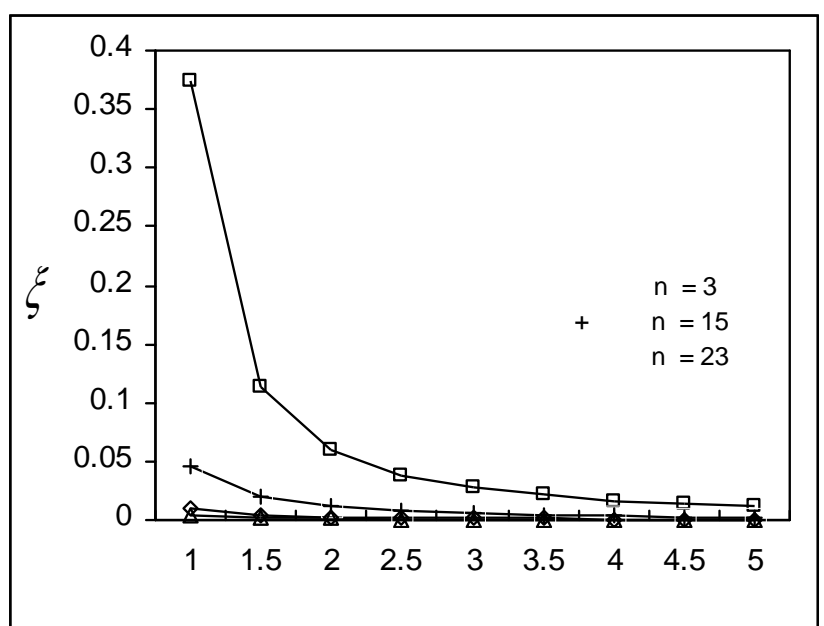

It is again discovered that the expected square of the difference of two sample medians rapidly diminishes with the increase in sample size for each $\beta$ value. 
Also, the quantity $\xi$ decreases rapidly when $\beta$ increases for the same sample size. From the elbow behavior we may consider the quasi median as a useful replacement for the sample median for the estimation of the Weibull median. However, for a small sample and for $\beta<1$ the use of quasi median does not seem reasonable.

\section{Correlation between Quasi and Sample Medians}

The first sample quasi median being nearest the sample median, it is useful for this study to include the correlation graphs for these medians

Fig 9

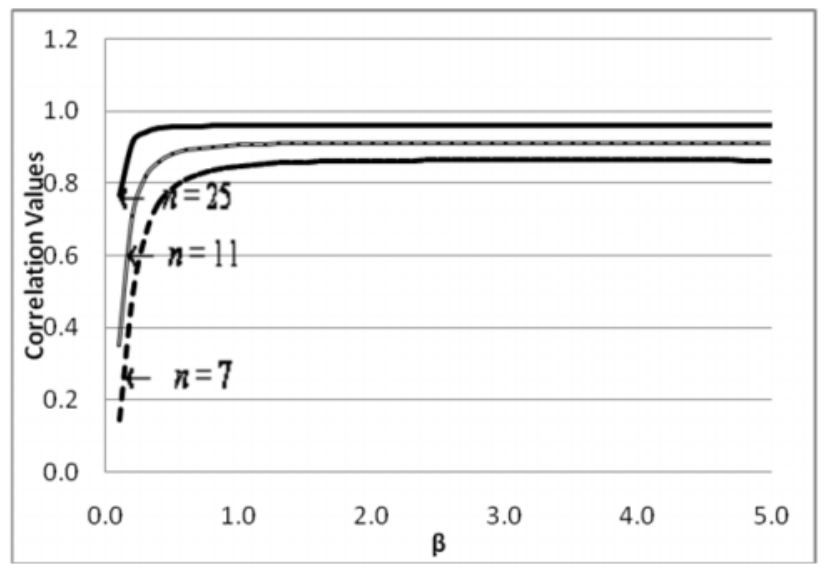

These graphs indicate a higher degree of correlation for larger samples. The Weibull parameter also influences this correlation; for $0<\beta<0.5$ the degree of correlation coefficient rapidly moves to an upper limit (the analysis here is confined to $\beta$ not beyond 5). As the sample size increases the correlation coefficient assumes a nearly constant value soon after $\beta<1$. A larger sample has a higher upper limit.

\section{Sample Range and Quasi Median}

It is known that for the Weibull random variable $X$ the $\operatorname{Prob}(X<c)$ increases with the increase in its parameter; $c$ being a fixed number. The expected value of the sample range for the same sample size also decreases as the value of $\beta$ increases. We have here graphs for $n=9,15,25$ showing the expected value of the sample range as well as for the bias caused by a sample quasi median at each value of $\beta$. By Memon (1987), or otherwise, it can be shown that the expected range for $\beta=1$ simplifies to

$$
\sum_{i=1}^{n-1}(1 / i)
$$

a result that also appears in Hiraii (2). 
Fig 10

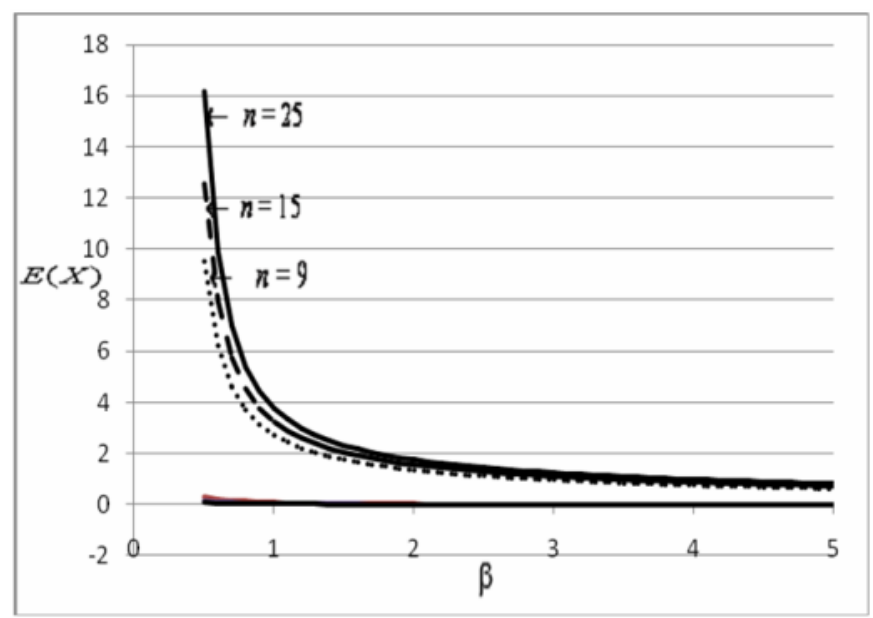

The amount of bias almost vanishes for $\beta>1$ even for smaller samples, as also already observed in the previous section. The interesting point is that even a large sample starts revealing an exponential decline in its sample ranges as $\beta$ increases.

\section{Conclusion}

The median of a population is normally estimated by its associated sample median. In the case of non-availability of necessary information to determine this median, one may consider the option of using quasi medians to estimate the population median. Our focus being on Weibull distribution, it is discovered in view of various criteria that the first quasi median becomes a reasonable substitute for the sample median when the sample size is not small, or when $\beta>1$.

\section{References}

1. Hirai, A.S. (1978). Moments of order statistics from the Raleigh distributions, Journal of Statistical Research. Vol. 12, No. 1 \& 2, University of Dacca, Bangladesh.

2. Hirai, A.S. (1998). A Course in Mathematical Statistics, llimi Kitab Khana.

3. Hodges, J.L. Jr. \& Lehmann, E.L. On Medians and Quasi Medians. Journal of the American Statistical Association. 62; 1967: 926-931.

4. Hogg, R.V \& Craig, A.T. (1978). Introduction to Mathematical Statistics, Macmillan Publishing Company, Inc. New York.

5. Memon, A.Z. \& Daghel M.H. (1987). Some studies of order statistics from a Weibull distribution, Pakistan Journal of Statistics, 3(1) A, 23-30.

6. Memon, A.Z. (2008). Some Remarks on Weibull Order Statistics. Pakistan Journal of Statistics 24 (1), $11-20$.

7. Weibull, W. (1951) A Statistical Distribution Function of Wide Applicability. J. App Mech., 18, 293-29. 


\section{APPENDIX}

TABLE - 1

EXPECTED VALUES

FIRST AND LAST SAMPLE QUASI MEDIANS

\begin{tabular}{|c|c|c|c|c|c|c|}
\hline \multirow{2}{*}{$\boldsymbol{\beta}$} & \multicolumn{3}{|c|}{$E\left(\mathbf{Z}_{(\mathbf{v})}\right)$} & \multicolumn{3}{c|}{$\boldsymbol{E}\left(\boldsymbol{Z}_{(1)}\right)$} \\
\cline { 2 - 7 } & $\mathbf{n = 7}$ & $\mathbf{n = 1 1}$ & $\mathbf{n = 2 5}$ & $\mathbf{n = 7}$ & $\mathbf{n}=\mathbf{1 1}$ & $\mathbf{n = 2 5}$ \\
\hline 0.5 & 0.9020 & 0.7015 & 0.5603 & 4.1378 & 5.3471 & 8.0852 \\
\hline 1.0 & 0.8012 & 0.7532 & 0.7160 & 1.3679 & 1.5554 & 1.9280 \\
\hline 1.5 & 0.8255 & 0.8067 & 0.7921 & 1.0453 & 1.1176 & 1.2599 \\
\hline 2.0 & 0.8510 & 0.8427 & 0.8364 & 0.9517 & 0.9854 & 1.0529 \\
\hline 2.5 & 0.8713 & 0.8678 & 0.8652 & 0.9173 & 0.9332 & 0.9663 \\
\hline 3.0 & 0.8871 & 0.8861 & 0.8854 & 0.9043 & 0.9108 & 0.9252 \\
\hline 3.5 & 0.8997 & 0.9000 & 0.9003 & 0.9004 & 0.9013 & 0.9048 \\
\hline 4.0 & 0.9098 & 0.9109 & 0.9118 & 0.9006 & 0.8982 & 0.8948 \\
\hline 4.5 & 0.9181 & 0.9197 & 0.9210 & 0.9028 & 0.8982 & 0.8905 \\
\hline 5.0 & 0.9250 & 0.9269 & 0.9284 & 0.9058 & 0.9000 & 0.8894 \\
\hline
\end{tabular}

TABLE - 2

EXPECTED VALUES

SAMPLE QUASI MEDIAN AND SAMPLE MEDIAN

\begin{tabular}{|c|c|c|c|c|c|}
\hline \multirow{2}{*}{$\beta$} & \multicolumn{2}{|c|}{$\mathrm{n}=\mathbf{3}$} & \multicolumn{2}{c|}{$\mathrm{n}=\mathbf{2 5}$} & \multirow{2}{*}{$\begin{array}{c}\boldsymbol{Z}_{M}= \\
(\mathbf{L}(\mathbf{2}))^{(1 / \beta)}\end{array}$} \\
\cline { 2 - 5 } & $E\left(\boldsymbol{Z}_{(\mathrm{v})}\right)$ & $E\left(\boldsymbol{Y}_{(\mathrm{v}+1)}\right)$ & $E\left(\boldsymbol{Z}_{(\mathrm{v})}\right)$ & $E\left(\boldsymbol{Y}_{(\mathrm{v}+1)}\right)$ & \\
\hline 0.5 & 2.4722 & 1.0556 & 0.5603 & 0.5488 & 0.4805 \\
\hline 1.0 & 1.0833 & 0.8333 & 0.7160 & 0.7127 & 0.6931 \\
\hline 1.5 & 0.9351 & 0.8381 & 0.7921 & 0.7909 & 0.7832 \\
\hline 2.0 & 0.9010 & 0.8566 & 0.8364 & 0.8358 & 0.8326 \\
\hline 2.5 & 0.8940 & 0.8738 & 0.8652 & 0.8650 & 0.8636 \\
\hline 3.0 & 0.8955 & 0.8880 & 0.8854 & 0.8853 & 0.8850 \\
\hline 3.5 & 0.8998 & 0.8996 & 0.9003 & 0.9004 & 0.9006 \\
\hline 4.0 & 0.9050 & 0.9091 & 0.9118 & 0.9119 & 0.9124 \\
\hline 4.5 & 0.9103 & 0.9171 & 0.9210 & 0.9211 & 0.9218 \\
\hline 5.0 & 0.9153 & 0.9238 & 0.9284 & 0.9285 & 0.9293 \\
\hline
\end{tabular}

TABLE - 3

BIAS

SAMPLE QUASI MEDIAN AND SAMPLE MEDIAN

\begin{tabular}{|c|c|c|c|}
\hline \multirow{2}{*}{$\beta$} & \multicolumn{3}{|c|}{$\tau=E\left(Y_{(v+1)}-Z_{(v)}\right)$} \\
\cline { 2 - 4 } & $\mathrm{n}=3$ & $\mathrm{n}=11$ & $\mathrm{n}=25$ \\
\hline 0.5 & -1.4167 & -0.0646 & -0.01151 \\
\hline 1.0 & -0.2500 & -0.0167 & -0.00321 \\
\hline 1.5 & -0.0970 & -0.0065 & -0.00126 \\
\hline 2.0 & -0.0444 & -0.0028 & -0.00054 \\
\hline 2.5 & -0.0202 & -0.0012 & -0.00022 \\
\hline 3.0 & -0.0075 & -0.0003 & -0.00006 \\
\hline 3.5 & -0.0003 & 0.0001 & 0.00003 \\
\hline 4.0 & 0.0041 & 0.0004 & 0.00007 \\
\hline 4.5 & 0.0068 & 0.0006 & 0.00010 \\
\hline 5.0 & 0.0085 & 0.0007 & 0.00012 \\
\hline
\end{tabular}


TABLE - 4

MEAN SQUARED ERROR

SAMPLE QUASI MEDIAN AND SAMPLE MEDIAN

\begin{tabular}{|c|c|c|c|c|}
\hline \multirow{2}{*}{$\beta$} & \multicolumn{2}{|c|}{$\mathbf{n = 3}$} & \multicolumn{2}{c|}{$\mathbf{n = 2 5}$} \\
\cline { 2 - 5 } & $\operatorname{MSE}\left(\mathbf{Z}_{(v)}\right)$ & $\operatorname{MSE}\left(Y_{(v+1)}\right)$ & $\operatorname{MSE}\left(Z_{(v)}\right)$ & $\operatorname{MSE}\left(Y_{(v+1)}\right)$ \\
\hline 0.5 & 13.6040 & 2.3405 & 0.1026 & 0.1055 \\
\hline 1.0 & -0.4455 & -0.2940 & 0.0386 & 0.0411 \\
\hline 1.5 & -0.6878 & -0.5316 & 0.0209 & 0.0224 \\
\hline 2.0 & -0.7257 & -0.6332 & 0.0131 & 0.0141 \\
\hline 2.5 & -0.7466 & -0.6954 & 0.0090 & 0.0097 \\
\hline 3.0 & -0.7647 & -0.7388 & 0.0066 & 0.0071 \\
\hline 3.5 & -0.7811 & -0.7712 & 0.0050 & 0.0054 \\
\hline 4.0 & -0.7961 & -0.7964 & 0.0039 & 0.0042 \\
\hline 4.5 & -0.8096 & -0.8166 & 0.0032 & 0.0034 \\
\hline 5.0 & -0.8216 & -0.8331 & 0.0026 & 0.0028 \\
\hline
\end{tabular}

TABLE - 5

$E\left(Y_{(v+1)}-Z_{(v)}\right)^{2}$

\begin{tabular}{|c|c|c|c|}
\hline \multirow{2}{*}{$\beta$} & \multicolumn{3}{|c|}{$\tau=E\left(Y_{(v+1)}-Z_{(v)}\right)^{2}$} \\
\cline { 2 - 4 } & $\mathbf{n = 3}$ & $\mathbf{n = 1 5}$ & $\mathbf{n = 2 3}$ \\
\hline 0.5 & 11.5417 & 0.0327 & 0.0110 \\
\hline 1.0 & 0.3750 & 0.0091 & 0.0038 \\
\hline 1.5 & 0.1143 & 0.0047 & 0.0020 \\
\hline 2.0 & 0.0595 & 0.0029 & 0.0013 \\
\hline 2.5 & 0.0384 & 0.0020 & 0.0009 \\
\hline 3.0 & 0.0276 & 0.0015 & 0.0006 \\
\hline 3.5 & 0.0211 & 0.0011 & 0.0005 \\
\hline 4.0 & 0.0168 & 0.0009 & 0.0004 \\
\hline 4.5 & 0.0138 & 0.0007 & 0.0003 \\
\hline 5.0 & 0.0116 & 0.0006 & 0.0003 \\
\hline
\end{tabular}

TABLE - 6

CORRELATION COEFFICIENTS

BETWEEN SAMPLE QUASI AND SAMPLE MEDIAN

\begin{tabular}{|c|c|c|c|}
\hline \multirow{2}{*}{$\beta$} & \multicolumn{3}{|c|}{ Correlation Values } \\
\cline { 2 - 4 } & $\mathbf{n = 7}$ & $\mathbf{n = 1 1}$ & $\mathbf{n}=\mathbf{2 5}$ \\
\hline 0.5 & 0.7865 & 0.8806 & 0.9551 \\
\hline 1.0 & 0.8482 & 0.9065 & 0.9598 \\
\hline 1.5 & 0.8595 & 0.9107 & 0.9605 \\
\hline 2.0 & 0.8628 & 0.9119 & 0.9606 \\
\hline 2.5 & 0.8638 & 0.9122 & 0.9607 \\
\hline 3.0 & 0.8641 & 0.9123 & 0.9607 \\
\hline 3.5 & 0.8640 & 0.9123 & 0.9606 \\
\hline 4.0 & 0.8638 & 0.9123 & 0.9607 \\
\hline 4.5 & 0.8636 & 0.9122 & 0.9607 \\
\hline 5.0 & 0.8634 & 0.9121 & 0.9612 \\
\hline
\end{tabular}


TABLE - 7

EXPECTED VALUES

\begin{tabular}{|c|c|c|c|c|c|c|}
\hline \multirow{2}{*}{$\beta$} & \multicolumn{2}{|c|}{$\mathrm{n}=9$} & \multicolumn{2}{c|}{$\mathrm{n}=15$} & \multicolumn{2}{c|}{$\mathrm{n}=25$} \\
\cline { 2 - 7 } & Range & Q.M & Range & Q.M & Range & Q.M \\
\hline 0.5 & 9.5181 & 0.7719 & 12.5822 & 0.6282 & 16.1641 & 0.5603 \\
\hline 1.0 & 2.7179 & 0.7706 & 3.2516 & 0.7343 & 3.7760 & 0.7160 \\
\hline 1.5 & 1.7522 & 0.8136 & 2.0435 & 0.7993 & 2.3086 & 0.7921 \\
\hline 2.0 & 1.3492 & 0.8457 & 1.5626 & 0.8395 & 1.7513 & 0.8364 \\
\hline 2.5 & 1.1150 & 0.8691 & 1.2897 & 0.8665 & 1.4429 & 0.8652 \\
\hline 3.0 & 0.9570 & 0.8865 & 1.1075 & 0.8857 & 1.2396 & 0.8854 \\
\hline 3.5 & 0.8412 & 0.8999 & 0.9746 & 0.9002 & 1.0922 & 0.9003 \\
\hline 4.0 & 0.7518 & 0.9105 & 0.8723 & 0.9114 & 0.9790 & 0.9118 \\
\hline 4.5 & 0.6804 & 0.9191 & 0.7905 & 0.9203 & 0.8885 & 0.9210 \\
\hline 5.0 & 0.6219 & 0.9262 & 0.7234 & 0.9276 & 0.8142 & 0.9284 \\
\hline
\end{tabular}

